\title{
Electrolyte Secretion Alteration
}

National Cancer Institute

\section{Source}

National Cancer Institute. Electrolyte Secretion Alteration. NCI Thesaurus. Code C41552.

A finding indicating a deficiency or excess in secretion of one or more electrolytes. 